\title{
Clarification-réflexion-régulation des valeurs
}

\section{Neus Sanmartí et Rosa Maria Tarin}

\section{(2) OpenEdition}

\section{Journals}

Édition électronique

URL : http://journals.openedition.org/ere/6937

DOI : $10.4000 /$ ere.6937

ISSN : 2561-2271

Éditeur

Centr'ERE

Référence électronique

Neus Sanmartí et Rosa Maria Tarin, "Clarification-réflexion-régulation des valeurs », Éducation relative à l'environnement [En ligne], Volume 2 | 2000, mis en ligne le 15 septembre 2000, consulté le 17 avril 2021. URL : http://journals.openedition.org/ere/6937 ; DOI : https://doi.org/10.4000/ere.6937

Ce document a été généré automatiquement le 17 avril 2021 


\title{
Clarification-réflexion-régulation des valeurs
}

\author{
Neus Sanmartí et Rosa Maria Tarin
}

1 Il paraît difficile de parler d'éducation relative à l'environnement sans adhérer en même temps à un certain système de valeurs (Fien, 1993). Mais est-il possible d'éduquer aux valeurs? Comment se construisent-elles? Est-ce que l'évaluation peut jouer un rôle dans cette construction?

2 Les termes «valeur» ou «éduquer» sont susceptibles de lectures multiples. Par exemple " éduquer » aux valeurs pourrait avoir diverses interprétations : endoctriner, instruire, habituer, former, enseigner, développer, etc.

3 Nous savons que les valeurs ne sont pas le résultat d'un savoir conscient. Dans la vie, nous agissons très rarement en adaptant nos comportements à nos façons de penser et d'évaluer. C'est plutôt le contraire : nous agissons d'une certaine façon et ensuite nous «inventons» des arguments pour justifier ces actions en faisant en sorte que les dissonances soient réduites.

4 Cependant, il existe une ressemblance surprenante entre les arguments employés par différents individus, ce qui laisse penser que chaque groupe social est porteur de certaines valeurs et des théories qui leur correspondent, même si souvent les unes et les autres sont implicites. Ces valeurs et ces théories façonnent ce que l'on a appelé une cosmovision, c'est-à-dire une vision et une interprétation globale du monde et de l'existence fondées sur des valeurs et des théories qui reflètent les intérêts du groupe social porteur. Il semble que nous adoptons une cosmovision, avec les valeurs et les théories qui la façonnent, parce que nous la saisissons dans le groupe social auquel nous appartenons.

5 Quelle serait donc la fonction de l'éducation, comprise comme une action consciente exercée par des éducateurs? Nous envisageons l'éducation comme un processus visant à favoriser, dans des situations d'interaction sociale, la prise de conscience et l'autorégulation des formes de penser, de promouvoir des valeurs et d'agir des différents membres d'un groupe (Tarin et Sanmartí, 1997). 
6 De notre point de vue, l'apprentissage de l'auto-évaluation est la composante majeure de cette façon de conceptualiser l'éducation relative à l'environnement. Il s'agit d'apprendre à «identifier » les implicites dans les formes d'agir et de penser (Sauvé, 1991), à les "analyser et à prendre des décisions" en fonction de cette analyse. Ce processus de clarification-réflexion-régulation est ce que l'on entend habituellement par évaluer. Toutefois, à partir de cette conceptualisation, le but de l'évaluation n'est pas de qualifier et classer les projets ou les personnes, mais de promouvoir leur autonomie par rapport à l'orientation de leur propre processus de changement.

7 Cet article examinera ces idées et plus particulièrement la fonction que peut jouer l'évaluation dans un processus de construction consciente des valeurs.

\section{L'évaluation en tant que processus de clarification- réflexion-régulation}

8 Selon les propos constructivistes et socio-critiques, l'évaluation est un élément intrinsèque de l'activité qui conduit à l'apprentissage, c'est-à-dire à bâtir de nouvelles façons de penser et d'agir (Sauvé, 1997).

9 Évaluer est un processus qui implique la collecte de données, l'analyse et la formulation de jugements, puis la prise de décisions en conséquence. À travers l'évaluation, les personnes peuvent reconnaître le degré de cohérence entre les idées explicites et les idées implicites, et entre les valeurs manifestes et les valeurs mises en pratique. L'analyse de ces données doit permettre à l'enseignant et aux élèves l'identification des raisons qui sont à l'origine des incohérences, des obstacles ou des difficultés que l'on devrait surmonter. Cela peut nous amener à la prise de décisions pour l'organisation de l'action de la façon la plus cohérente et efficace possible en fonction des objectifs fixés, et peut même mettre en cause ces objectifs.

Cette acception de l'évaluation est totalement différente de celle qui ne vise qu'à connaître les résultats d'une action éducative, même si le processus est similaire. Dans ce dernier cas, l'évaluation est fondamentalement associée à l'activité, elle se fait à la fin de l'action et elle a pour but de recueillir des données concernant les différences entre les objectifs initiaux et les objectifs atteints, de façon à pouvoir prendre des décisions visant à classer les élèves, les enseignants ou les programmes avec une finalité sélective ou d'amélioration du projet éducatif. En revanche, les finalités d'une évaluation «formatrice » (Nunziati, 1990) comme celle que nous poursuivons sont très différentes.

11 Derrière chaque façon de concevoir l'évaluation existent des valeurs implicites auxquelles correspondent certaines idéologies. C'est pourquoi, comme le disent Losito et Mayer (1997), «le type d'évaluation doit être cohérent avec l'éthique environnementale soutenue ».

12 Ainsi, dans le domaine de l'éducation relative à l'environnement, il est intéressant d'étudier spécialement les formes d'évaluation basées sur l'équité et la promotion de la solidarité, l'autonomie, la valorisation et l'accueil de tous les points de vue (tolérance) et la responsabilité dans l'apprentissage collectif. Cela se concrétise par une évaluation qui soit auto-évaluation et coévaluation entre les personnes d'un collectif dont le but est de coopérer à l'autorégulation consciente des formes d'expliquer et d'agir en fonction d'une finalité commune. 
13 Mais, à partir de cette perspective, que faut-il évaluer concernant les valeurs et à quelle fin? Quels sont les aspects qu'il est important d'évaluer? Qui évalue ? Comment mener à bien cette évaluation-régulation?

\section{Peut-on évaluer les valeurs séparément des cosmovisions dans lesquelles elles se trouvent insérées?}

Les problèmes environnementaux sont complexes; il est difficile d'avoir une connaissance scientifique globale qui en expliquerait les causes et permettrait d'envisager les conséquences. Chacun peut donc construire sa propre représentation des causes et des solutions. Cette représentation influence l'expression des idées et les pratiques, la conduite individuelle et la conduite collective.

Les représentations se basent davantage sur le système de valeurs construit par chaque individu et chaque groupe social que sur l'application de critères rationnels fondés sur des connaissances scientifiques ou d'un autre ordre. Or, l'éducation relative à l'environnement est précisément basée sur des valeurs. Les valeurs sont, selon Goffin (1997), des « entités conceptuelles pourvues d'une force symbolique, qui lorsqu'elles se concrétisent par des actions réelles, confèrent aux acteurs d'une certaine communauté culturelle une légitimité et une estime sociale ». En fait, les valeurs expriment des souhaits, des façons de vivre individuellement et socialement préférables aux valeurs contraires, même si elles n'ont pas encore été atteintes.

Le rôle des valeurs dans la configuration des idées environnementales et de l'action est très important. Une personne, par exemple, peut être d'avis que l'utilisation de papier recyclé n'a pas de sens, une autre peut penser le contraire. L'une et l'autre peuvent apporter des arguments en faveur de leurs opinions. La personne qui est contre l'utilisation du papier recyclé donne des arguments du genre: les propriétaires des forêts doivent les entretenir. Il leur faut donc de l'argent; leurs revenus les plus importants sont ceux qui dérivent de la transformation du bois en pâte à papier; ou, les eaux résiduelles produites par le recyclage du papier sont fortement polluées par les teintures qu'elles contiennent ; ou encore, le papier recyclé a une couleur grisâtre, etc. En revanche, la personne qui prend parti pour l'utilisation du papier recyclé argumente : il est important de ne pas consommer de matières premières si cela n'est pas nécessaire ; il faut tenir compte de l'impact environnemental, du cycle complet du papier et non seulement d'une partie de ce cycle ; elle évoque la beauté du gris dans le Guernica de Picasso, etc.

Les uns et les autres donnent des arguments scientifiques, sociaux, économiques, esthétiques, etc. relatifs à leurs différentes cosmovisions, étroitement liés au système de valeurs personnel. Ce système de valeurs justifie pour chaque personne la validité des arguments. C'est ainsi que les informations reçues seront incorporées au système cognitif d'une personne comme valables ou non.

18 Nous constaterons cette vision dans une recherche effectuée. Nous avons proposé la figure 1 et le commentaire ci-dessous à des élèves de 9-11 ans, après avoir étudié le cycle urbain de l'eau, pour évaluer comment ils avaient intégré les connaissances travaillées en classe (Sanmartí et Tarin, 1991). 
Il s'agit d'un village de montagne où il n'y a pas encore l'eau courante. Près du village se trouve une rivière et en amont une usine qui traite le bois et pollue l'eau de la rivière. Imagine tout ce qu'il faudrait faire pour mettre en place un système de distribution d'eau qui ferait arriver l'eau potable à toutes les maisons du village à partir de l'eau de cette rivière.

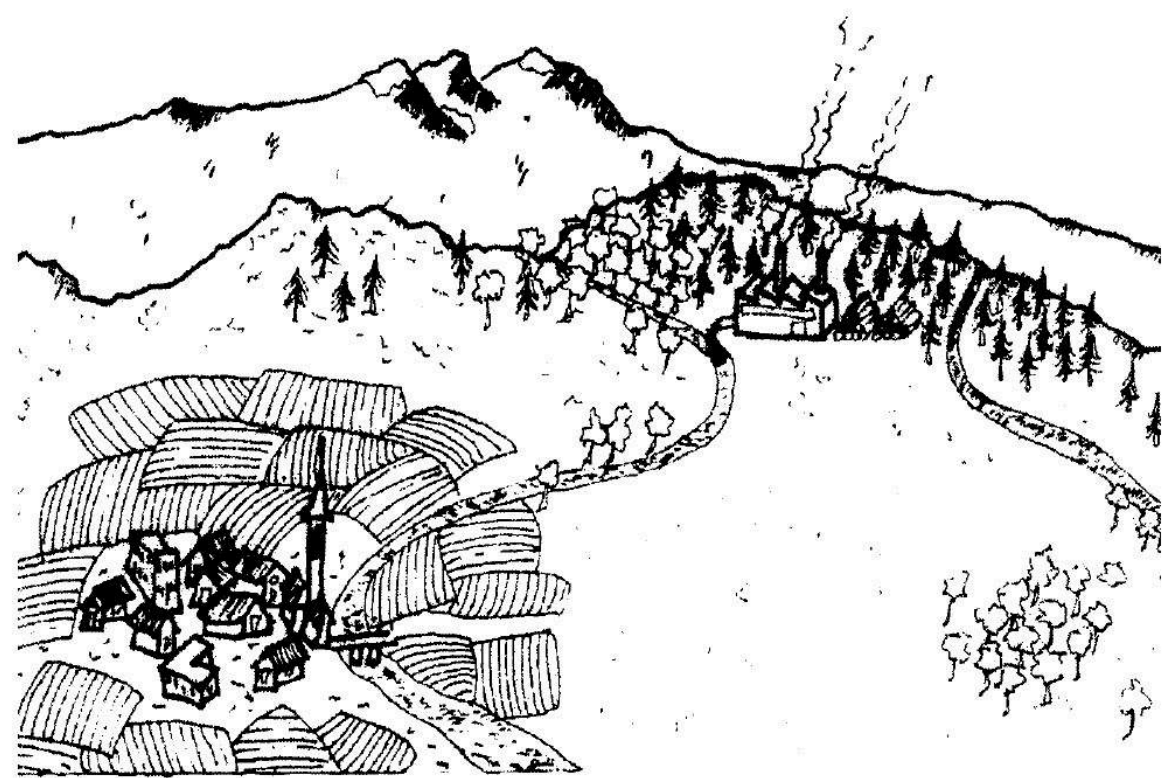

Figure 1 : Exercice «Village de montagne»

Dans la figure 2, l'élève décide de supprimer l'usine, et donc d'éliminer le problème de la pollution. Il s'agit d'une solution que l'on pourrait considérer comme conservatrice et simple, dans laquelle ont été prises en compte les causes du problème, mais pas les conséquences socio-économiques liées à la suppression de l'usine. La représentation « conserver sans changer » possède des valeurs associées.

Dans la figure 3, par contre, l'élève décide de placer l'usine ailleurs, de façon à ce qu'elle pollue d'autres environnements et non pas celui qui lui est proche. Nous observons que cette solution ne contient pas la valeur « solidarité à l'échelle non locale». 


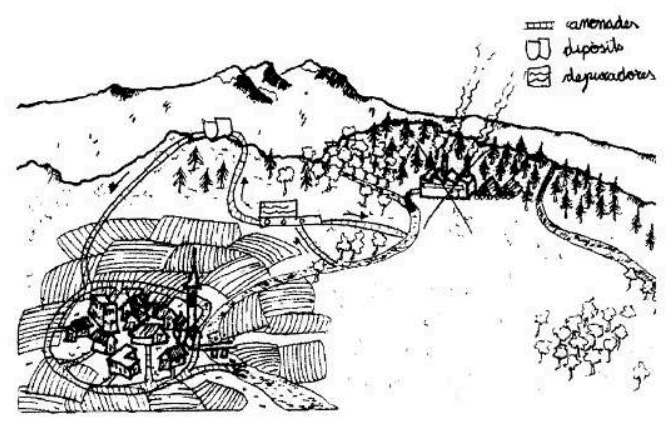

Figure $2: \propto$ Village de montagne ", réponse 1

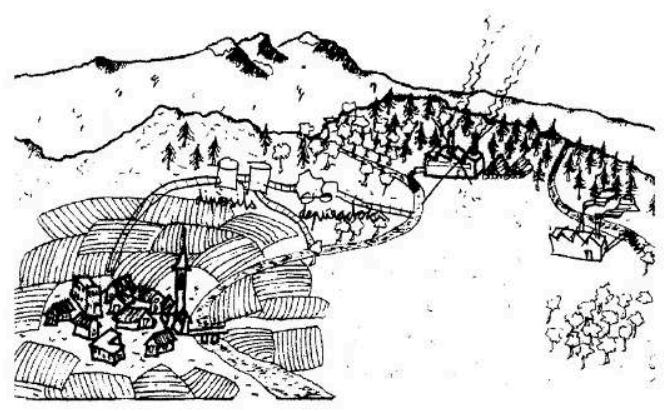

Figure $3:$ « Village de montagne $*$, réponse 2

Dans la figure 4, l'élève propose une solution compatible avec des modèles technologiques de développement durable.

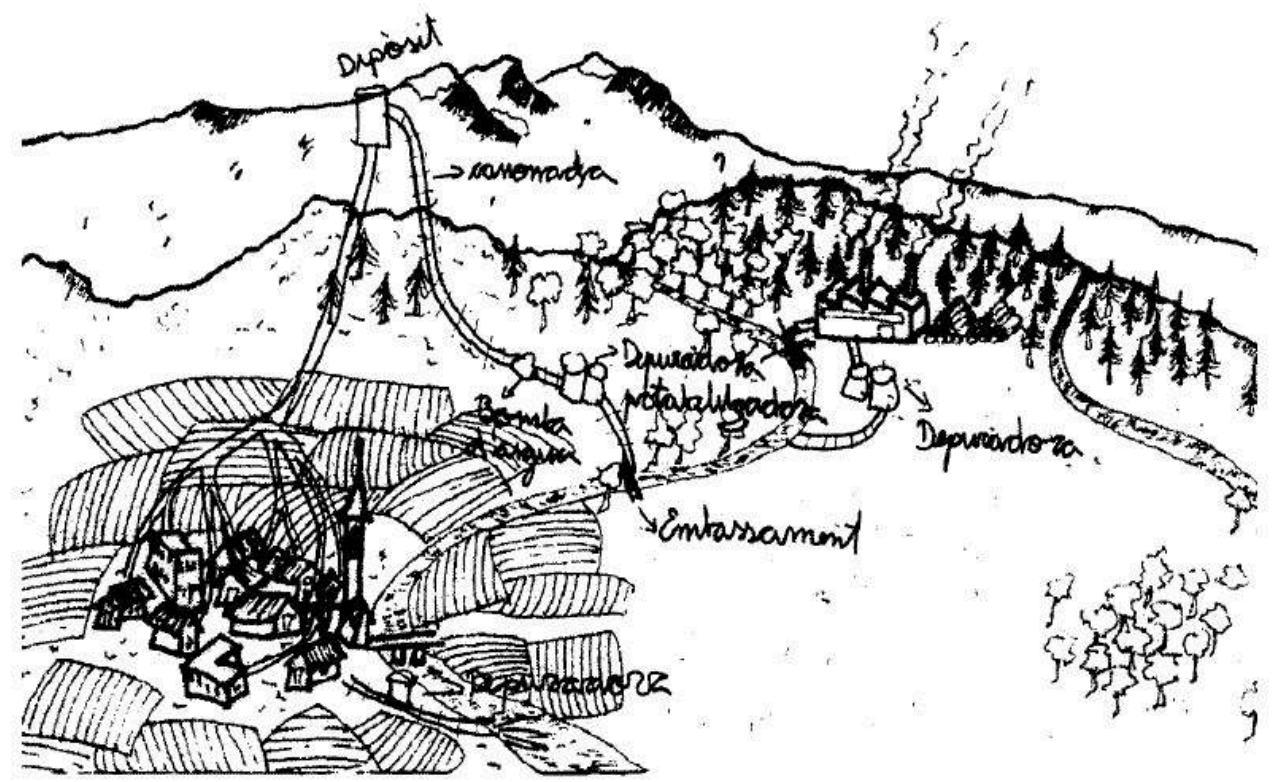

Figure $4:$ : Village de montagne », réponse 3

Les trois conceptions reflètent des cosmovisions différentes dans lesquelles sont intégrés des systèmes de valeurs et des connaissances. Ce genre d'exercice donne la possibilité d'évaluer les valeurs implicites dans la proposition de solutions - prise de décisions concernant un problème environnemental. Les valeurs n'ont pas été explicitement sollicitées dans la question. Mais les réponses les ont mises en évidence. 
Les représentations des causes et des solutions des problèmes environnementaux sont étroitement liées au système de valeurs des personnes et des groupes sociaux. Il n'existe pas une lecture objective des faits ; toute lecture est interprétative et implique des jugements de valeurs et des prises de décisions. Plutôt que d'essayer de juger des valeurs isolées, il sera donc important de reconnaître le système de valeurs implicite dans la représentation d'un problème environnemental ou dans une action.

Par exemple, l'évaluation de l'exercice «village de montagne » a une fonction surtout formative, puisqu'elle permet de réfléchir sur les différents points de vue et de discuter à propos des valeurs qui déterminent les idées et les actions.

L'évaluation du discours sur les valeurs nous oblige donc aussi à identifier celles qui sont sous-jacentes dans une représentation, les analyser, et prendre des décisions, c'est-à-dire réguler. Chaque représentation comprend des valeurs de ce qui est souhaitable, beau, possible, utile, adéquat, nécessaire, etc. Comme il a été indiqué, les évaluer signifie les mettre au grand jour, les examiner et mettre en évidence les incohérences entre le système des valeurs déclarées et celui des valeurs mises en pratique. Évaluer signifie surtout réguler, c'est-à-dire planifier des changements si cela devient nécessaire.

27 Par conséquent, ce qui importe n'est pas d'évaluer les comportements, mais plutôt de relever les inconsistances éventuelles et les dissonances (Riechmann, 1999), réfléchir à propos du fonctionnement de ces actions - leur « logique » - et très spécialement, viser à ce que les élèves apprennent à réaliser ce processus de clarification-réflexionrégulation de façon autonome. Aider à construire un certain schéma axiologique implique de développer l'autonomie dans le jugement moral et cela est en rapport étroit avec l'apprentissage de l'auto-évaluation et de l'autorégulation.

Le texte encadré, ci-dessous, est une reproduction partielle d'une activité proposée dans le but de relever d'éventuelles divergences entre les valeurs déclarées et celles, souvent plus réelles, relatives à l'acceptation ou la transgression des règles habituelles implicitement établies (Torras, 1995). Il existe généralement un consensus sur ce que l'on admet socialement comme étant une activité «correcte» du point de vue de l'environnement et la majorité des personnes répond selon ces schémas établis aux questions de la première section du questionnaire. Ce n'est qu'en posant des questions qui échappent à ces schémas (comme, par exemple, celles de la deuxième section) que l'on favorise l'identification de valeurs implicites et leur analyse, de même que leur possible autorégulation. 
Sommes-nous cohérents?

1. La municipalité de XXX propose une série d'actions. Quel est ton avis concernant ces actions?

- Installer des conteneurs pour les objets en verre afin que tous les citoyens les y déposent.

- Porter plainte contre les entreprises qui polluent l'eau systématiquement.

- Encourager l'usage du transport public face au privé par l'offre de tickets de voyage gratuits.

- Interdire aux supermarchés d'offrir des sacs en plastique à leurs clients.

2. Outre ces actions la municipalité en a proposé d'autres comme celles qui suivent. Quel est ton avis à leur propos?

- Faire payer uen amende de 10,000 dollars à toute personne qui jette un objet en verre dans le conteneur d'ordures au lieu de le jeter dans le conteneur de verre.

- Porter plainte contre toute personne qui prend systématiquement un bain au lieu de prendre une douche.

- Encourager l'alimentation méditerranéenne par l'imposition d'impôts aux aliments préfabriqués (pâtisserie industrielle, hamburgers, etc.) de façon à ce que le prix de ces produits se multiplie par 100.

- Interdire l'achat d'une voiture neuve aux personnes possédant une voiture de moins de 8 ans.

3. Discuter les valeurs implicites dans les opinions exprimées, à partir de la réflexion spécialement axée sur les causes d'éventuelles différences d'avis entre 1 et 2.

\section{Évaluer suppose une clarification des valeurs. Mais quelles valeurs?} durabilité ", il existe au moins deux visions différentes possibles (Sauvé, 1998). L'une d'elles vise à éduquer pour produire et consommer rationnellement, sans que le modèle économique et social dans lequel nous produisons et nous consommons soit mis en cause. L'autre vision nécessite un changement du modèle d'organisation sociale et de vie pour produire et consommer moins. La cosmovision et les valeurs implicites dans chacune de ces visions sont très différentes et nous pouvons déduire de cette diversité des actions éducatives très différentes.

Par exemple, face au problème de la pollution des eaux, une première vision peut promouvoir essentiellement des formes de rationalisation de la consommation et de l'épuration. Une seconde nous amènerait à tenir compte de ce que plus on consomme de biens (voiture, papier, etc.), plus on pollue les eaux. Adhérer à cette deuxième vision suppose promouvoir des "styles de vie» différents de ceux qui sont en général valorisés dans les sociétés développées.

Chaque groupe social a des valeurs dominantes issues d'une éthique dominante. Par exemple, on pourrait affirmer que "conserver l'eau» est un objectif consensuel largement accepté dans notre société occidentale (pas pour autant pratiqué), mais l'objectif de « changer le style de vie de la société de consommation » est perçu comme quelque chose qui ne peut être imposé, qui est lié à la diversité des choix personnels, 
idéologiques, politiques, etc. En ce sens, personne ne doute de la nécessité de traiter le premier objectif à l'école, tandis que l'introduction du second serait perçue comme endoctrinement. Il est cependant possible que ces appréciations changent, au fil du temps, en fonction des variations des valeurs collectives dominantes.

Nous pouvons, en outre, nous demander s'il existe des valeurs immuables et universelles. La crise de valeurs de la société postmoderne a été un sujet de discussion dans le passé et elle l'est encore actuellement. Néanmoins, nous pouvons nous interroger : "au-delà des options qui se présentent actuellement dans les sociétés démocratiques et complexes est-il possible de penser rationnellement qu'il existe des critères que tout le monde pourrait considérer comme étant souhaitables, sans avoir à revenir sur des modèles à valeurs absolues?» (Puig, 1995), c'est-à-dire des principes qui pourraient être considérés comme la base d'une pensée démocratique.

Quels seraient ces principes universels? Est-il possible d'établir une hiérarchie de valeurs?

Différents auteurs situent l'équité comme le principe de base et le point de départ de toute réflexion éthique concernant l'environnement (Novo, 1995 ; Pujol, 1998). L'équité, entendue comme « la responsabilité morale d'aider à la croissance des autres à partir de leurs propres capacités et conditionnements, de façon différenciée", se fonde sur « la conviction profonde qu'il n'existe d'injustice majeure que celle de traiter comme égaux les inégaux » (Novo, 1995, p. 91). Par conséquent, cette valeur serait supérieure à d'autres valeurs consensuelles telles l'égalité et la liberté. Notons que les bénéfices du comportement écologiquement conscient sont rarement individuels, mais plutôt collectifs. De même, l'augmentation du bien collectif entraîne une diminution du bien individuel. C'est pour tout cela que le principe éthique de l'équité est mis en rapport avec les cosmovisions et avec des idéologies alternatives par rapport à celles actuellement prédominantes dans les sociétés occidentales développées.

Étroitement lié à ce principe d'équité, il existe aussi un certain consensus pour considérer la solidarité appliquée à l'environnement comme une valeur fondamentale, entendue comme «l'adoption de solutions aux problèmes environnementaux compte tenu tant des besoins de la société humaine dans son ensemble que des priorités de certains groupes sociaux s'imposant aux autres " (Novo, 1995, p. 92). La solidarité est mise en rapport avec l'intérêt porté aux besoins des collectivités humaines, avec l'intérêt porté aux besoins considérés à l'échelle sociale et à l'échelle planétaire, avec les générations actuelles et avec les générations futures.

Goffin (1997) établit quatre valeurs essentielles qui, en fait, sont interdépendantes: Solidarité, Tolérance, Autonomie et Responsabilité (STAR). Cette dernière, la responsabilité, couronne toutes les autres et doit être comprise comme l'engagement des personnes et des collectivités vis-à-vis de l'impact que leurs actions ont sur l'environnement. Dans cette même direction, Sauvé (1998) propose, quand il s'agit de donner un nom aux différentes propositions éducatives de type transversal qui ont en commun l'encouragement d'une éthique de la responsabilité, de parler «d'éducation pour le développement des sociétés responsables ».

Bien qu'il semble qu'un certain accord existe actuellement pour définir ces valeurs comme essentielles et universelles, tout le monde ne les concrétise pas de la même façon. Il est possible de prendre les valeurs STAR comme points de référence, mais il semble difficile de les définir de manière univoque. 
Reprenons l'exemple des arguments pour ou contre la consommation de papier recyclé. Les personnes qui défendent les points de vue décrits plus haut peuvent considérer que les valeurs qui sous-tendent leurs idées et leur façon d'agir sont la solidarité, l'autonomie et la responsabilité. En conséquence, ces valeurs ne doivent pas s'identifier de façon isolée, il est important de les mettre en relation avec les différentes façons d'expliquer et d'évaluer les faits du monde, qui sont intégrés dans ce que l'on appelle la cosmovision.

Changer la façon d'analyser, d'expliquer et d'agir par rapport à la problématique environnementale entraîne un changement dans les valeurs individuelles et collectives. Mais est-il possible d'apprendre à le faire?

\section{Quels sont les aspects importants à évaluer?}

41 Pour que l'évaluation favorise le développement de l'autonomie, elle devrait aider à la clarification de ce qui détermine une action ou une activité de la personne. L'évaluation est alors une expression de la capacité des acteurs d'apprendre dans l'action, de développer un savoir pratique aussi efficace qu'efficient, pour organiser l'action de la façon la plus adéquate en fonction des objectifs visés. Nous pourrions affirmer que l'évaluation est surtout et avant tout, une composante intrinsèque de l'action qui conduit à produire des changements (Zúñiga, 1994).

Le but de toute action éducative est la construction rationnelle et autonome de valeurs dans des situations d'interaction sociale. Ce processus de construction souhaitable exige l'identification de valeurs personnelles et d'autrui, l'analyse et la formulation de jugements et la prise de décisions. Il nécessite une progression dans un processus d'évaluation de type métacognitif (Milà et Sanmartí, 1999). La rhétorique, comprise comme la capacité de persuasion des éducateurs et des membres du groupe, et leurs rapports affectifs mutuels ont une forte influence dans ce processus. La rationalité peut aider à prendre conscience du rôle de la rhétorique et des sentiments dans l'adhésion à certaines valeurs (Camps, 1998).

Dans cette perspective, il est important d'évaluer les implicites des actions à partir de l'analyse des discours en général et plus précisément à partir de l'étude des arguments élaborés pour justifier nos manières d'agir face aux autres. Toute action vise, en fonction de certains critères, l'atteinte d'objectifs et implique une anticipation et une planification des opérations à réaliser, ainsi qu'une reconnaissance des critères qui donnent la possibilité d'identifier la qualité des résultats obtenus (Nunziati, 1990). L'évaluation devrait s'orienter fondamentalement vers la clarification-réflexionrégulation des valeurs implicites en rapport avec les façons de penser, d'agir et de sentir, car elles limitent :

- les motifs de l'activité, de l'action (réelle ou simulée) : pourquoi ? Quels arguments?

- les objectifs : pour quoi faire ? À quelle fin ? Que veut-on obtenir?

- la planification du processus de la pensée-action: les fondements du processus? Quels arguments? Quelles conditions-limitations ont été imposées? Parmi les stratégies possibles, quelle est celle qui a été choisie? Comment s'organise le groupe ? Comment sont gérés les conflits? etc.

- les critères d'évaluation: qu'espérons-nous atteindre? Quels sont les aspects qui sont relevés, et quels aspects ne le sont pas? Comment sont justifiés les résultats? 
L'exercice décrit dans l'encadré reproduit une tâche qui vise à apprendre à évaluer les valeurs implicites dans la justification de projets d'action alternatifs (15-16 ans). Cette tâche favorise l'identification des valeurs lors de l'analyse des arguments tant par rapport aux motifs de l'action que par rapport aux critères utilisés pour évaluer sa pertinence, son appréciation lors de la comparaison des différents points de vue et finalement la prise de décisions lors de l'explicitation des nouveaux points de vue possibles (ou de leur réfutation).

"Jouons aux journalistes "
La classe est divisée en deux groupes d'élèves. On demande aux membres de l'un de ces
groupes d'écrire individuellement un article pour un journal local qui soutiendra un
projet d'élargissement de l'aéroport au détriment d'une réserve naturelle. Chacun des
membres du deuxième groupe devra écrire un article pour la conservation et l'améliora-
tion de la même réserve naturelle.
Un échange d'articles entre les membres de groupes différents aura ensuite lieu et chacun
évaluera les articles à l'aide des indications suivantes :
- Quelles sont les valeurs contenues dans les motivations de chaque projet et dans leur
vision du progrès?
- Quelles valeurs peuvent être reconnues dans les critères utilisés pour évaluer les béné-
fices-cồts éventuels de chacun des plans d'action proposés? Penser à ceux qui concer-
nent l'économie, l'environnement, la qualité de vie, etc. au niveau individuel ou col-
lectif, à court terme ou à long terme.
Après l'échange de perceptions de l'ensemble du groupe, on distribue deux articles parus
dans les journaux soutenant les deux points de vue discutés. Puis on compare les argu-
ments de la presse avec ceux qui sont issus du groupe-classe. Un débat est alors introduit
par la question suivante:
À la lecture de ce genre d'articles, la majorité reconnaît celui qui correspond le mieux à
ses idées mais elle ne fait rien pour les défendre. Quelles valeurs implicites peuvent être
identifiées dans ce type de comportement?
Pour conclure, on demande à chaque individu d'écrire, de manière anonyme, une
réflexion à propos des changements possibles de sa manière d'évaluer les articles ou les
nouvelles de ce genre (prise de décisions). Ses opinions sont distribuées afin de les faire
connaître à l'ensemble du groupe.

Lorsque nous avons réalisé ce type d'activités en classe, nous avons observé que, la première fois, seuls certains étudiants étaient capables d'exprimer verbalement les valeurs qui ont été signalées comme "essentielles » et les contre-valeurs. Mais, en fonction des caractéristiques du groupe, et tout spécialement de celles des personnes qui exercent un rôle hégémonique (parmi lesquelles l'enseignant), le processus évaluatif des valeurs implicites dans la résolution de certains problèmes est transformé - tel que l'indique Zúñiga (1994) - en un processus de changement: ce qui semblait «normal » devient problématique.

\section{Qui fait l'évaluation?}

Quant à l'évaluation elle-même, objet de cette analyse, le processus de clarificationréflexion-régulation est personnel et il doit être mené à bien par chaque individu. Néanmoins, cela n'est possible que dans la mesure où les personnes font partie d'un groupe qui favorise l'expression des différents points de vue.

C'est pour cela que nous parlons d'auto-évaluation et de coévaluation, qui ne peuvent exister que si le groupe admet que toutes les idées sont dignes d'être écoutées et soutenues, que la pluralité de visions est quelque chose de légitime, de souhaitable même, etc. 
48 Le rôle de l'éducateur est complexe puisqu'il doit aussi bien manifester son appréciation, son jugement, que promouvoir une ambiance et un système de travail qui favorisent l'expression de tous les points de vue, le sien et celui des autres. « Il s'agit de croire en ce que l'on fait et laisser la place à d'autres croyances » (Mayer, 1998). Ce qui est donc important ce n'est pas d'encourager l'acceptation aveugle de certaines valeurs, toutes écologistes qu'elles soient, mais de discuter les différents propos.

Nous pourrions affirmer que, pour une institution éducative, l'objectif prioritaire est d'expliciter les valeurs, de les détailler et de les discuter, plutôt que de parvenir à ce que tous leurs membres adhèrent à des valeurs déterminées. Pour éviter l'endoctrinement, il est essentiel de comprendre que la tolérance et le respect de toutes les opinions peuvent être un cadre qui impose des limites à la persuasion et à la belligérance.

50 Si nous reprenons l'exercice « village de montagne » (figures 1 à 4), les élèves, à travers leurs opinions, ne manifestent pas seulement leurs connaissances, mais aussi leurs valeurs implicites. Le rôle de l'enseignant ne sera plus celui de sanctionner telle ou telle réponse, mais plutôt de faciliter la discussion sur les valeurs implicites. La prise de décisions - ou régulation - concerne les élèves eux-mêmes et ils devront l'exercer en pleine autonomie.

51 Le respect des opinions n'est pas un obstacle à la discussion et ne remet pas en doute certains points de vue, il ne contraint pas à accepter tous les comportements et autorise tout établissement éducatif à promouvoir certaines pratiques. Dans des situations d'incertitude, nous avons tendance à agir comme le font les autres. Tel est le pouvoir du bon exemple.

52 Dans ce processus de clarification, il est essentiel de ne pas oublier d'autres facteurs qui influencent aussi la prise de décision. Par exemple, l'hégémonie affective exercée par les représentants de certaines options. Une étude (Cunill et coll., 1992) sur l'expression des causes et des solutions aux problèmes environnementaux par des élèves de 12 à 16 ans dans six établissements scolaires de différents niveaux socio-culturels, relève que les opinions et les valeurs exprimées étaient relativement semblables malgré la diversité sociale (voir encadré). 


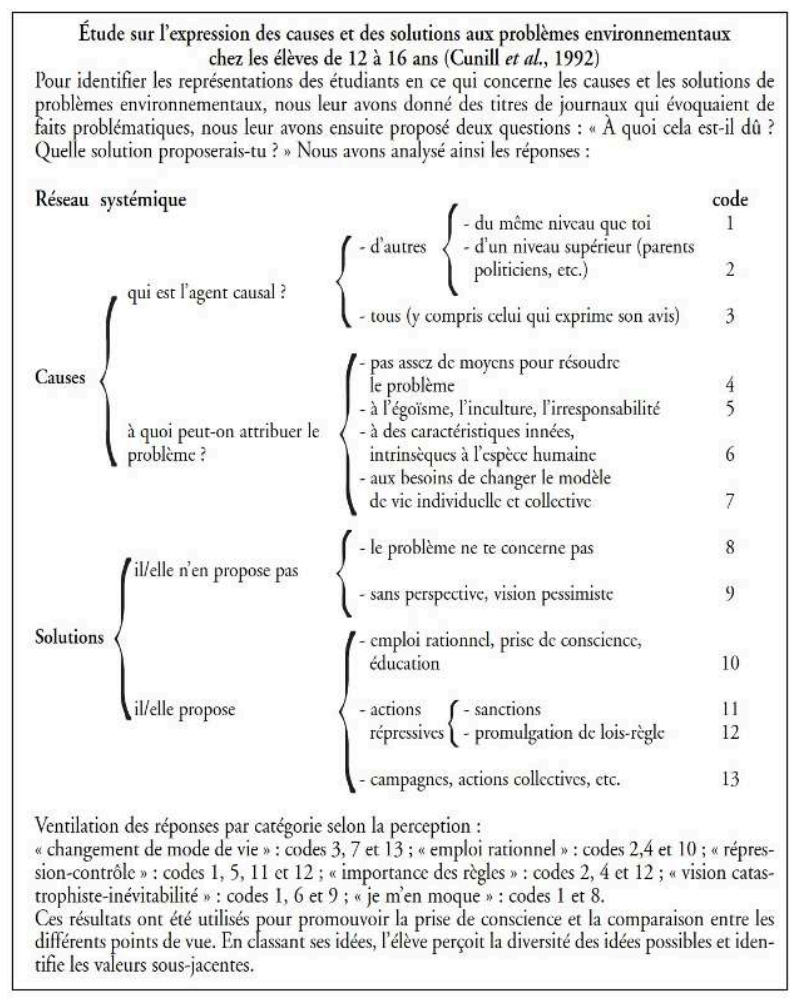

53 On observe, en revanche, des résultats très différents dans une classe d'un de ces établissements. D'après les enseignants, cette divergence serait expliquée par la présence d'une fille aux idées très "écologistes" et leader très appréciée de ses camarades (Maymó, 1994).

Mais même dans le cas d'un leader influent, l'identification des valeurs sous-jacentes sera à encourager. Les valeurs (et les contre-valeurs) s'apprennent parce que nous les saisissons par le fait d'être membre d'un groupe social qui les proclame, les justifie et les met en pratique. Chacun doit les construire en interaction avec les autres.

\section{Conclusion}

Nous avons essayé de relever la nécessité de promouvoir, dans le domaine de l'éducation relative à l'environnement, l'apprentissage de l'auto-évaluation, comprise comme un processus de clarification-réflexion-régulation du système de valeurs implicites qui oriente les actions de toute personne et de tout groupe social.

Cet apprentissage implique la nécessité d'apprendre à auto évaluée les valeurs qui soustendent les motifs, les objectifs, la planification et les critères de l'évaluation des actions. L'apprentissage ne sera mené à bien qu'au sein d'un groupe où les différentes options peuvent être manifestées librement et où l'espace et le temps permettent de les clarifier, d'y réfléchir et de prendre des décisions, sachant que cet apprentissage ne devient significatif que lorsqu'il prend appui sur l'action. Cela n'a aucun sens d'évoquer d'autres valeurs sans changer de pratiques.

Nous parlons donc d'une évaluation dans le dialogue, une évaluation qui cherche à développer un jugement moral fondé sur une rationalité émanant d'un dialogue et sur la capacité d'agir de façon autonome et cohérente. Il se peut que pour certains il s'agisse d'une évaluation utopique, aussi utopique que l'éducation dans le domaine des 
valeurs. Cependant, nous croyons que l'enseignement et l'apprentissage méritent d'être au centre de nos défis s'il s'agit de la formation d'êtres humains critiques.

\section{BIBLIOGRAPHIE}

Camps, V. (1998). La escuela ante el reto del saber práctico. Infancia y Aprendizaje, 82, 65-73.

Clary, M. (1997). Les particularités de l'évaluation en ErE. Environnement \& Société, 19, 33-42.

Cunill, M.A., Melià, R. et Ferrés, C. (1992). Avaluació d'una campanya d'EA. Univ. Autònoma de Barcelona. No publicado.

Fien, J. (1993). Critical Curriculum Theorizing in Environmental Education. Geelong : Deakin University Press.

Goffin, L. (1997). L'éducation relative à l'environnement, un défi pour le XXIe siècle. Conférence forum international Planèt' ErE, Montréal.

Losito, B. et Mayer, M. (1997). Une évaluation cohérente avec les valeurs et les principes de l'ErE. Environnement \& Société, 19, 43-52.

Mayer, M. (1998). Educación Ambiental : De la acción a la investigación. Enseñanza de las Ciencias, 16(2), 217-231.

Maymó, S. (1994). Anàlisi de les idees dels estudiants d'Educació Secundària sobre què és un problema ambiental. Treball Magister. Univ. Autònoma de Barcelona. No publicado.

Milà, C. et Sanmartí, N. (1999). A Model for Fostering the Transfer of Learning in Environmental Education. Environmetal Education Research, 5 (3), 237-266.

Novo, M. (1995). La Educación Ambiental. Bases éticas, conceptuales y metodológicas. Madrid: Ed. Universitas.

Nunziati, G. (1990). Pour construire un dispositif d'évaluation formatrice. Cahiers pédagogiques, 280, 47-64.

Puig, J.M. (1995). Les possibilitats de la crisi de valors. Vela Major, 2, 37-41.

Pujol, R.M. (1998). Modelos de integración de la Educación del Consumidor en el currículo escolar : Un análisis desde el área de Ciencias. Tesis doctoral. Univ. Autònoma de Barcelona. No publicado.

Riechmann, J. (1999). Inconsistencias, disonancias y bloqueos : atisbos sociopsicológicos sobre la crisis ecológica. Dans J. Riechmann, J. Sempere et A. Izquierdo (dir.), Todo tiene un límite. Sociología y medio ambiente. Madrid: Ed. Síntesis.

Sanmartí, N. et Tarin, R.M. (1991). L'educació ambiental, una necessitat de formació actual ? Butlletí de Mestres, 229, 9-14.

Sauvé, L. (1991). La clarification des valeurs. L'enjeu, 11 (4), 21-22.

Sauvé, L. (1997). Pour une approche critique de l'évaluation en ErE. Environnement \& Société, 19, 19-26. 
Sauvé, L. (1998). L'éducation relative à l'environnement : Entre modernité et postmodernité. Les propositions du développement durable et de l'avenir viable. Colloque la Voie verte. Web d'Environnement Canada.

Tarin, R.M. et Sanmartí, N. (1997). L'apprentissage et la régulation à travers des situations problèmes. Environnement \& Société, 19, 59-66.

Torras, A. (1995). Actituds de respecte : Abast i significats de l'expressió. Treball Magister. Univ. Autònoma de Barcelona. No publicado.

Zúñiga, R. (1994). Planifier et évaluer l'action sociale. Montréal : Presses de l'Université de Montréal.

\section{RÉSUMÉS}

Cet article tente d'examiner le rôle de l'apprentissage de l'auto-évaluation dans le processus de construction des valeurs dans le domaine de l'éducation relative à l'environnement. En prenant appui sur des exemples d'activités réalisées en classe, le texte insiste sur la nécessité d'apprendre à clarifier les rapports entre les représentations des causes et des solutions des problèmes environnementaux et leurs valeurs implicites, d'apprendre à réfléchir sur les dissonances et incohérences éventuelles entre les valeurs proclamées et les valeurs pratiquées, ainsi qu'à expliciter les aspects à réguler et planifier des changements si cela devient nécessaire.

This article aims to examine the role of the learning process of self-evaluation in the construction process of values within the domain of environmental education. Based on examples of classroom activities, the text insists on the necessity of learning to clarify the relations between the representations of causes and solutions to environmental problems and their implicit values, of learning to reflect on the dissonance and possible incoherence between proclaimed and practised values, as well as on the necessity of learning to explicit the aspects to regulate and to plan changes when it becomes necessary.

\section{AUTEURS}

\section{NEUS SANMARTÍ}

Neus Sanmartí détient un doctorat en chimie et professeure à l'Université Autonome de Barcelona (UAB). Elle a travaillé dans le domaine d'ErE et de la régulation métacognitive par sa participation à de nombreux projets espagnols et européens, en donnant des conférences, des cours et en participant à des séminaires. Elle est l'auteure d'outils didactiques, d'articles en ERE et dirige des travaux de recherche sur ce thème. Elle est coordinatrice du GREDA (Groupe de recherche en ErE) de l'UAB.

\section{ROSA MARIA TARIN}

Rosa Maria Tarin est licenciée en chimie, professeure à l'Université Autonome de Barcelona (UAB). Dans le domaine d'ErE, elle a plus particulièrement étudié les processus d'évaluation et de régulation des apprentissages, mais également la coéducation, l'interculturalité et l'apprentissage des valeurs en sciences. Elle est coauteur de plusieurs outils didactiques, de projets et a écrit plusieurs articles dans ces domaines. Elle est membre du GREDA (Groupe de recherche en ErE) de l'UAB. 